\title{
NOTE
}

\section{The Moisturizing Effects of Glycolipid Biosurfactants, Mannosylerythritol Lipids, on Human Skin}

\author{
Shuhei Yamamoto', Tomotake Morita ${ }^{2}$, Tokuma Fukuoka², Tomohiro Imura², \\ Shusaku Yanagidani ${ }^{1}$, Atsushi Sogabe ${ }^{3}$, Dai Kitamoto ${ }^{2}$ and Masaru Kitagawa ${ }^{3 *}$ \\ ${ }^{1}$ Toyobo Co., Ltd. Tsuruga Institute of Biotechnology (10-24, Toyo-cho, Tsuruga, Fukui, 914-0047, Japan) \\ ${ }^{2}$ Research Institute for Innovations in Sustainable Chemistry, National Institute of Advanced Industrial Science and Technology (AIST) (5-2 \\ Tsukuba Central, 1-1 Higashi, Tsukuba, Ibaraki 305-8565, Japan) \\ ${ }^{3}$ Toyobo Co., Ltd. Biochemical Dept. (2-8-8,Dojima Hama, Kita-Ku, Osaka, 530-8230, Japan)
}

\begin{abstract}
Glycolipid biosurfactants, such as mannosylerythritol lipids (MELs), are produced by different yeasts belonging to the genus Pseudozyma and have been attracting much attention as new cosmetic ingredients owing to their unique liquid-crystal-forming and moisturizing properties. In this study, the effects of different MEL derivatives on the skin were evaluated in detail using a three-dimensional cultured human skin model and an in vivo human study. The skin cells were cultured and treated with sodium dodecyl sulfate (SDS), and the effects of different lipids on the SDS-damaged cells were evaluated on the basis of cell viability. Most MEL derivatives efficiently recovered the viability of the cells and showed high recovery rates (over $80 \%$ ) comparable with that of natural ceramide. It is interesting that the recovery rate with MEL-A prepared from olive oil was significantly higher than that of MEL-A prepared from soybean oil. The water retention properties of MEL-B were further investigated on human forearm skin in a preliminary study. Compared with the control, the aqueous solution of MEL-B (5 wt\%) was estimated to considerably increase the stratum corneum water content in the skin. Moreover, perspiration on the skin surface was clearly suppressed by treatment with the MEL-B solution. These results suggest that MELs are likely to exhibit a high moisturizing action, by assisting the barrier function of the skin. Accordingly, the yeast glycolipids have a strong potential as a new ingredient for skin care products.
\end{abstract}

Key words: moisturizing activity, glycolipid, mannosylerythritol lipids, ceramide, three-dimensional cultured skin model, damaged human skin

\section{INTRODUCTION}

Skin roughening, mainly due to dryness, is generally caused by damage to the intracellular lipids of the skin, which decreases the water-retention capacity of the stratum corneum. Thus, highly moisturizing agents, such as ceramides, which are considered to assist the water-retention function of the $\operatorname{skin}^{1}{ }^{1}$, have been receiving increasing attention as an effective ingredient for skin care cosmetics and pharmaceuticals ${ }^{2)}$.

While natural ceramides have distinctive moisturizing properties, the amount of ceramide produced by each organism is very limited; thus, the large-scale preparation of these natural lipids is considerably difficult. Synthetic ceramides are obtained by acylation of the amine group of sphinganine or its derivative and are used in moisturizers as alternatives to natural ceramides. However, these synthetic approaches for the large-scale preparation of isomerically pure ceramides are tedious, time-consuming, and thus expensive to produce for commercial applications ${ }^{3)}$. It is thus of great interest to develop a new and cost-effective natural moisturizer alternative to ceramides.

Mannosylerythritol lipids (MELs, Fig. 1) are one of the most promising glycolipid biosurfactants, and are abundantly produced at a yield of over $100 \mathrm{~g} / \mathrm{L}$ from vegetable oils by different yeasts belonging to the genus Pseudozy$m a^{4,5)}$. Pseudozyma antarctica T-34 mainly produces MEL-A(Fig. 1a), whereas P. tsukubaensis NBRC1940 predominately produces the diastereomer type of MEL-B

\footnotetext{
*Correspondence to: Masaru Kitagawa, Toyobo Co., Ltd. Biochemical Department, 2-2-8, Dojima Hama, Kita-Ku, Osaka, 530-8230, Japan

E-mail: Masaru_Kitagawa@ toyobo.jp

Accepted February 8, 2012 (received for review November 13, 2011)

Journal of Oleo Science ISSN 1345-8957 print / ISSN 1347-3352 online

http://www.jstage.jst.go.jp/browse/jos/ http://mc.manusriptcentral.com/jjocs
} 
a

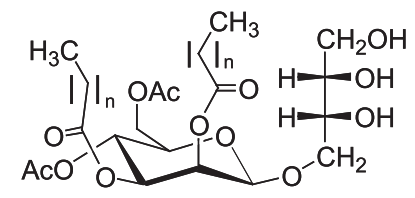

MEL-A

Pseudozyama antarctica T-34

$(n=8-12)$ b

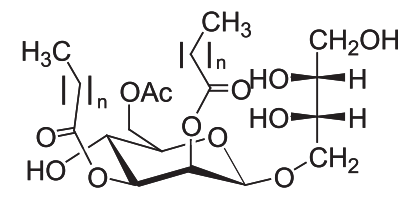

MEL-B

Pseudozyama tsukubaensis NBRC1940 $(n=8-14)$
C

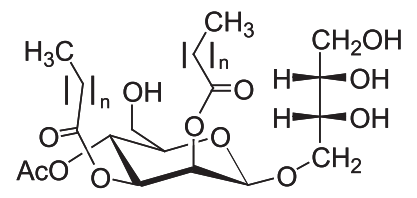

MEL-C

Pseudozyama hubeiensis KM-59

$(n=6-16)$

Fig. 1 Chemical Structure of MEL-A (a), MEL-B (b), and MEL-C (c).

(Fig. 1b). In addition, P. hubeiensis KM-59 mainly produces MEL-C (Fig. 1c). These MEL derivatives form different lyotropic liquid crystalline phases efficiently, including a lamellar $\left(\mathrm{L}_{\alpha}\right)$, in a broad range of concentrations ${ }^{6-8)}$. We have also previously demonstrated that MEL-A produced from soybean oil by $P$. antarctica T-34 shows potential moisturizing activity comparable with that of natural ceramide, based on an assay using the three-dimensional cultured human skin model ${ }^{9}$.

To broaden the application of MELs, we focused our attention on the detailed characterization of their effects on the skin. In this study, we investigated the effects of different MEL derivatives on damaged skin cells using a threedimensional cultured human skin model. We further evaluated the effect of MELs on the water retention properties of the skin, using an in vivo human study. Herein, we demonstrated for the first time that MELs have not only a strong effect on the recovery of damaged skin cells but also a potential moisturizing effect on the skin.

\section{EXPERIMENTAL}

\subsection{Production of MEL}

Based on our previous studies ${ }^{5)}$, P. antarctica T-34, P. tsukubaensis $\mathrm{NBRC} 1940^{\mathrm{T}}$, and $P$. hubeiensis KM-59 were used to obtain MEL-A, MEL-B, and MEL-C, respectively. These strains were cultured in liquid growth medium ( $4 \%$ glucose, $0.3 \% \mathrm{NaNO}_{3}, 0.03 \% \mathrm{MgSO}_{4}, 0.03 \% \mathrm{KH}_{2} \mathrm{PO}_{4}$, and $0.1 \%$ yeast extract $[\mathrm{pH} 6.0]$ ) at $25^{\circ} \mathrm{C}$ on a reciprocal shaker (150 strokes/min) for $2 \mathrm{~d}^{10)}$. The seed culture obtained $(0.1$ $\mathrm{mL}$ ) was transferred to a 200-mL Erlenmeyer flask containing $20 \mathrm{~mL}$ of a production medium $(5 \%(v / v)$ vegetable oil, $0.3 \% \mathrm{NaNO}_{3}, 0.03 \% \mathrm{MgSO}_{4}, 0.03 \% \mathrm{KH}_{2} \mathrm{PO}_{4}$, and $0.1 \%$ yeast extract $[\mathrm{pH} 6.0])$ at $25^{\circ} \mathrm{C}$ on a rotary shaker $(250$ rpm) for $7 \mathrm{~d}$. The glycolipids produced were extracted from the culture medium with an equal amount of ethyl acetate.

\subsection{Purification of MEL}

The upper organic layer was separated and evaporated. The concentrated MELs were dissolved in chloroform and then purified by silica gel (Wako-gel C-200; Wako, Japan) column chromatography using a gradient elution of chloro- form-acetone (10:0 to 0:10, $v / v)$ mixtures as solvent systems $^{10)}$. The purified MEL-A, MEL-B, and MEL-C fractions were used in the following experiments.

\subsection{Quantification of MEL by high-performance liquid chromatography}

The quantification of MEL was carried out by high-performance liquid chromatography (HPLC) on a silica gel column (100 A, $5 \mu \mathrm{m}, 4.6 \times 250 \mathrm{~mm}$; Inertsil SIL, GL Sciences Inc, Japan), with a low-temperature evaporative light-scattering detector (ELSD-LT; Shimadzu, Japan) using a gradient solvent program consisting of various ratios of chloroform to methanol (from 100:0 to 0:100, $v / v$ ) at a flow rate of $1 \mathrm{~mL} / \mathrm{min}$. The quantification of each MEL was based on the standard curve using the purified MEL fractions as described previously ${ }^{10}$.

\subsection{Structural analysis of MEL}

The structure of the purified MEL was confirmed by ${ }^{1} \mathrm{H}$ nuclear magnetic resonance with a Varian Inova-400 (400 $\mathrm{MHz}$ ) at $30^{\circ} \mathrm{C}$, using the $\mathrm{CD}_{3} \mathrm{OD}$ solution. The fatty acid profile of the purified MEL was analyzed by gas chromatography-mass spectrometry (Hewlett Packard 6890 and $5973 \mathrm{~N}$ ) with a TC-WAX (GL Sciences Inc) with a temperature program of $90^{\circ} \mathrm{C}$ held for $3 \mathrm{~min}$ and then rising to $240^{\circ} \mathrm{C}$ by $5^{\circ} \mathrm{C} / \mathrm{min}$, as described previously ${ }^{11)}$.

\subsection{Viability assay}

The potential effects of MELs on the skin were evaluated using a three-dimensional cultured human skin model (Testskin, Toyobo, Japan), where the readout was cell viability, as reported previously ${ }^{9)}$. An aqueous solution of 1 wt\% sodium dodecyl sulfate(SDS) was applied to the cell surface and incubated for $5 \mathrm{~min}$ at $37^{\circ} \mathrm{C}$ to induce damage similar to dry skin conditions. After washing 3 times with fresh culture medium $(1 \mathrm{~mL})$, olive oil fractions $(50 \mu \mathrm{L})$ containing various concentrations of lipids were directly applied, and then the cells were incubated for 24 hours at $37^{\circ} \mathrm{C}$. Natural ceramide III (of $94.4 \%$ purity) was purchased from Evonik Industries AG(Essen, Germany) and used as the positive control. The viability of the cells was colorimetrically determined using an MTT assay kit (Nakalai, Japan). The cells cultured without the SDS treatment were 
used as the control.

\subsection{Water content and perspiration at the surface of hu- man skin}

As a preliminary study, the water content and perspiration at the skin surface were estimated by a Corneometer (CM825, Integral, Japan) and a Tewameter (TM300, Integral, Japan), respectively ${ }^{12,13)}$. The forearm skin of 2 healthy female volunteers in their 30s was gently wiped with a cotton ball soaked with a soap solution, and rinsed with water. After drying with a paper towel, the volunteers were kept at $25^{\circ} \mathrm{C}$ and $50 \%$ humidity for 45 min. MEL-B was dissolved at a 1 or $5 \mathrm{wt} \%$ concentration into water containing 5\% 1,3-butylene glycol(BG). These solutions were applied to the soap-treated area of the forearm at $0.05 \mathrm{~mL} /$ area (approximately $2 \mu \mathrm{L} / \mathrm{cm}^{2}$ ). For both measurements, the area treated with only water was used as the control. The water content and perspiration were measured periodically, and the measurements were carried out at least 3 times in each area. The values were expressed as the mean of triplicate measurements, of which the experimental error was less than $10 \%$.

\section{RESULTS AND DISCUSSION}

\subsection{The skin care-related effects of MEL derivatives on} the cultured human skin cells

To estimate the skin care-related effects of different MEL derivatives on the skin, MEL-A, MEL-B, and MEL-C were prepared from olive oil and purified by silica gel column chromatography. The MEL-A(OL, Fig. 1a), MEL-B (OL, Fig. 1b), and MEL-C (OL, Fig. 1c) obtained from olive oil possessed $28.7 \%, 35.1 \%$, and $25.4 \%$ unsaturated fatty acids, respectively (Table 1). For comparison of fatty acid profile, MEL-A was also prepared from soybean oil; this MEL-A (SB) possessed 42.4\% unsaturated fatty acids.

These MEL derivatives were dissolved in olive oil at final concentrations of 1 and 5 wt \% and applied to the cultured human skin cells treated with SDS (Fig. 2). Natural ceramide III at 1 wt \% was used as the positive control. Olive oil alone had little effect on cell viability. In contrast, the ceramide clearly enhanced the recovery of the cells from SDS-induced damage, yielding high cell viability of over $90 \%$.

It is interesting that MEL-A (OL) yielded a cell viability of higher than 90\%, whereas MEL-A (SB) yielded significantly lower cell viability. Thus, the difference in the fatty acid composition, especially in the content of unsaturated fatty acids, may affect cell recovery owing to the difference in the self-assembling manner of the $2 \mathrm{MELs}^{7)}$. MEL-B (OL) also had a strong recovery effect on the SDS-damaged cells, and the cell viability reached over $80 \%$. MEL-C (OL) recovered the cell viability, but the recovery rate was lower than those by MEL-A(OL) and MEL-B (OL) .

All the MEL derivatives tested clearly had a recovery effect on the SDS-damaged skin cells, and the observed recovery rates were comparable with that of ceramide III. These results suggest that MELs have ceramide-like skin care properties. The recovery effect of the MEL derivatives on the damaged cultured human skin cells is probably due to the moisturizing activity, considering the well-known

Table 1 The fatty acid profiles of MELs.

\begin{tabular}{ccccc}
\hline \multirow{2}{*}{ Fatty acid } & \multicolumn{4}{c}{ Composition (\%) } \\
\cline { 2 - 5 } & MEL-A(SB) & MEL-A(OL) & MEL-B (OL) & MEL-C(OL) \\
\hline $6: 0$ & 0.0 & 0.0 & 0.7 & 21.2 \\
$8: 0$ & 27.3 & 22.5 & 38.3 & 2.6 \\
$8: 1$ & 3.3 & 1.9 & 0.1 & 0.2 \\
$10: 0$ & 21.3 & 41.4 & 3.5 & 19.4 \\
$10: 1$ & 27.2 & 26.3 & 1.2 & 0.1 \\
$10: 2$ & 5.4 & 0.5 & 0.0 & 0.0 \\
$12: 0$ & 4.7 & 7.4 & 20.1 & 16.7 \\
$12: 1$ & 4.8 & 0.0 & 0.0 & 3.3 \\
$12: 2$ & 0.0 & 0.0 & 0.0 & 5.2 \\
$14: 0$ & 0.0 & 0.0 & 2.4 & 4.8 \\
$14: 1$ & 0.0 & 0.0 & 21.9 & 14.6 \\
$14: 2$ & 1.7 & 0.0 & 11.9 & 4.1 \\
$16: 0$ & 0.0 & 0.0 & 0.0 & 0.0 \\
$16: 1$ & 0.0 & 0.0 & 0.0 & 1.1 \\
$16: 2$ & 0.0 & 0.0 & 0.0 & 2.1 \\
Unknown & 4.3 & 0.0 & 0.0 & 4.8 \\
\hline Unsaturated fatty acids & 42.4 & 28.7 & 35.1 & 25.4 \\
\hline
\end{tabular}




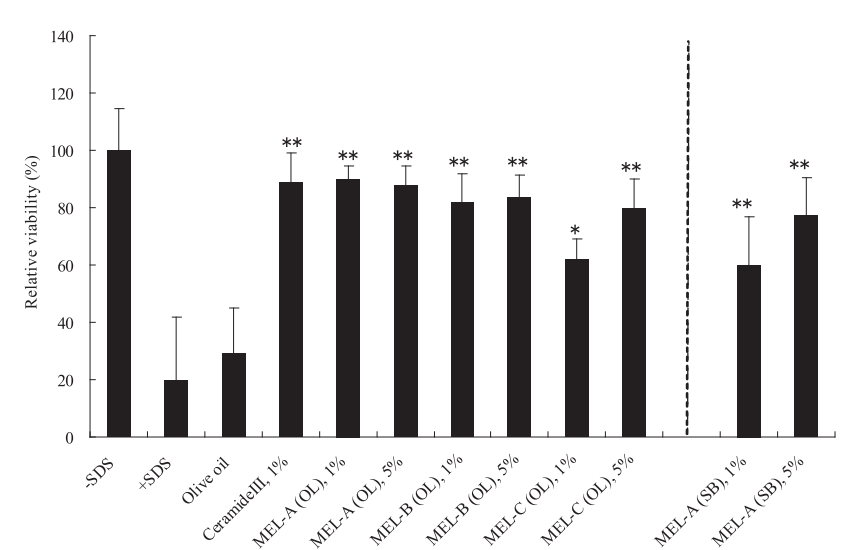

Fig. 2 Relative Viability of the Dry-damaged Skin Cell Model Treated with MEL derivatives.

The cultured skin cells were treated with $1 \%$ SDS, washed out the SDS solution, and then re-treated with MEL derivatives dissolved in olive oil. The viability of cells was determined with a colorimetric method (MTT assay) at 570 $\mathrm{nm}$. Ceramide was used as the positive control. -SDS: non-treated with SDS, +SDS: treated with SDS. Vertical bars show the standard error of the mean based on three independent measurements. Asterisk denotes a statistically significant difference from treated with SDS(Student's $t$ test; $* P<0.05, * * P<0.01)$

skin care properties of ceramides. Skin ceramide influences the water permeability properties of the skin, providing an epidermal water barrier, which strengthens the skin structure and reduces water loss. Regarding the mechanism of the recovery from damage, we also speculate that the MELs effectively facilitated moisture retention in the cells owing to the excellent formation of a lamella phase similar to ceramide ${ }^{7)}$.

\subsection{Water-retention properties of MEL-B in human skin}

The MEL-B (OL) produced in the present study by $P$. tsukubaensis was the diastereomer of conventional MEL-B produced by other strains of Pseudozyma and was reported to efficiently form a lamellar phase in a wide range of concentrations, compared with other MEL derivatives ${ }^{14)}$. The phase formed by intracellular lipids in the stratum corneum plays a crucial role in the barrier function of the $\operatorname{skin}^{15)}$. In addition, we recently accomplished the highyield production of the MEL-B from vegetable oil by a newly isolated strain of $P$. tsukubaensis, $1 \mathrm{E}-5$; the yield reached over $70 \mathrm{~g} / \mathrm{L}^{16)}$. To investigate the potential commercial application of MEL-B, we investigated its moisturizing properties in an in vivo human study.

MEL-B (OL) was dissolved in water containing $5 \%$ BG, which is a common humectant and solubilizer used in cos-

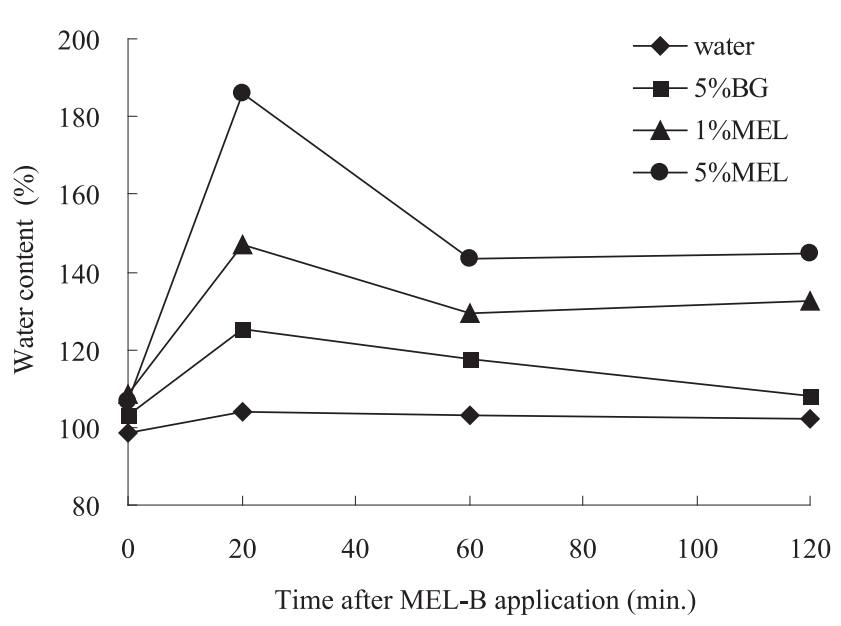

Fig. 3 Content of Water in Stratum Corneum Treated with the Diastereomer Type of MEL-B

The forearm skin was gently washed with a soap solution, and then re-treated with the MEL-B solution. The water-content capacity of the stratum corneum was measured by the corneometer. The value of water content after soap wash was taken as $100 \%$.

metic and food preparations. The MEL-B solution was applied to forearm skin and then the water-retention capacity of the stratum corneum was measured using a Corneometer (Fig. 3). The water content of the area treated with only $5 \%$ BG solution increased by up to $125 \%$. An interesting finding is that on the areas treated with the MEL-B solution, the increase in water content occurred in a dose-dependent manner. After 20 min of administration, the MEL-B solutions of 1 and $5 \mathrm{wt} \%$ yielded high water contents of $147 \%$ and $186 \%$, respectively (Fig. 3). Moreover, the positive effect of the MEL-B solution on the water content continued for the period of at least 2 hours.

\subsection{Suppression of human skin perspiration by MEL-B}

The effect of MEL-B on the barrier function of the human skin was also evaluated based on the measurement of skin perspiration with a Tewameter(Fig. 4). Skin perspiration was expressed in transepidermal water loss (TEWL) ${ }^{13}$, and the area treated with only water was used as the control. The area treated with only 5\% BG solution showed a minor increase in TEWL. It is interesting that on the areas treated with the MEL-B solution, a decrease in TEWL was observed and occurred in a dose-dependent manner. After 20 min of administration, the areas treated with the MEL-B solutions of 1 and $5 \mathrm{wt} \%$ yielded TEWLs of $86 \%$ and $74 \%$, respectively. More significantly, the areas treated with the MEL-B solution maintained lower TEWL values as compared with water and the BG solution throughout the 2 hours.

Based on the well-known moisturizing properties of ce- 


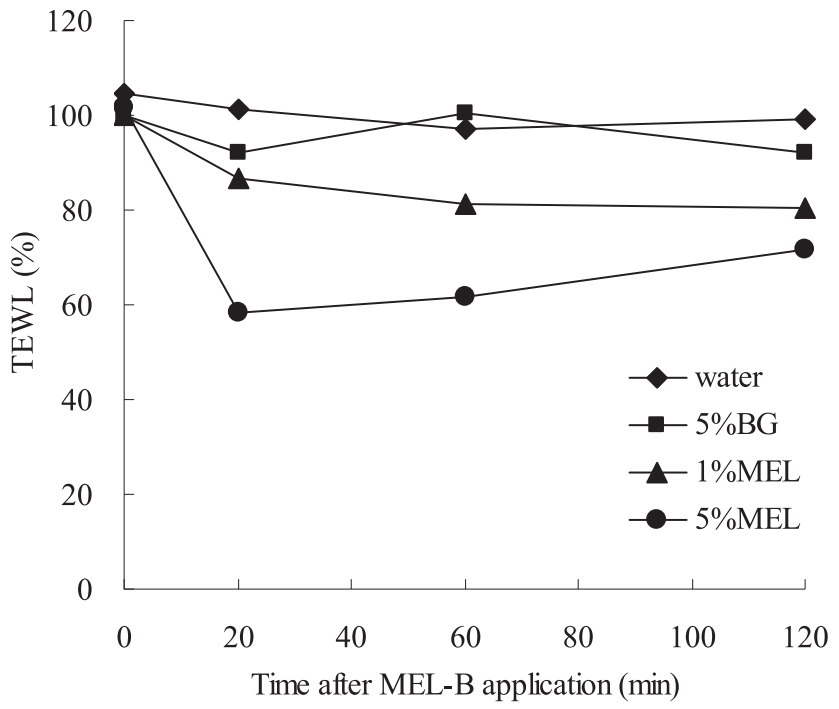

Fig. 4 Perspiration in Stratum Corneum Treated with the Diastereomer Type of MEL-B

The forearm skin was gently washed with a soap solution, and then re-treated with the MEL-B solution. The perspiration of the stratum corneum was measured by the tewameter. The value of water content after soap wash was taken as $100 \%$.

ramide ${ }^{17,18)}$, the MEL derivatives described in the present study are likely to play an important role in maintaining the water permeability of the skin and to assist in the maintenance of the epidermal water barrier, which strengthens the skin structure and reduces water loss. From these results, the glycolipids described, especially MEL-B, have a strong potential as a new moisturizing agent for human skin.

\section{CONCLUSION}

The moisturizing properties of different MEL derivatives, MEL-A, MEL-B, and MEL-C, were evaluated using a threedimensional cultured human skin model and an in vivo human study. All the MEL derivatives had a high recovery effect on the SDS-damaged skin cells. The observed recovery rates of the MELs were comparable with that of natural ceramide III. MEL-A prepared from soybean oil had a lower recovery effect on the cells compared with that prepared from olive oil, indicating that the fatty acid profile apparently affects moisturizing capacity. On the forearm skin, MEL-B not only increased the water content of the skin but also suppressed perspiration, and these positive effects continued for a period of at least 2 hours.

Ceramides have been receiving increasing attention as an effective moisturizing ingredient for cosmetics and pharmaceuticals owing to their potential water-holding properties ${ }^{19)}$. However, the commercial applications of ceramides are limited because of their extremely low water solubility; this makes the handling very difficult. On the other hand, MEL-B produced by P. tsukubaensis was reported to show higher hydrophilicity compared to other conventional MELs due to its unique configuration of the erythritol moiety ${ }^{20)}$. These facts suggest that MEL-B produced as described in the current study is highly advantageous not only for use in water-in-oil-type emulsifiers but also as an effective skin care integrant.

\section{Acknowledgments}

The authors thank Ms. C. Sano and Ms. E. Ito in AIST for their technical assistances.

\section{References}

1) Imokawa, G.; Akasaki, S.; Minematsu, Y.; Kawai, M. Importance of intracellular lipids in water-retention properties of the stratum corneum: induction and recovery study of surfactant dry skin. Arch. Dermatol. Res. 281, 45-51 (1989).

2) Mizutani, Y.; Mitsutake, S.; Tsuji, K.; Kihara, A.; Igarashi, $\mathrm{Y}$. Ceramide biosynthesis in keratinocyte and its role in skin function. Biochimie. 91, 784-790 (2009).

3) Elimelech, R. Process for large-scale preparation of sphingosines and ceramides. US6469148B1 (2002).

4) Kitamoto, D.; Morita, T.; Fukuoka, T.; Konishi, M.; Imura, T. Self-assembling properties of glycolipid biosurfactants and their potential applications. Curr. Opin. Colloid Interface Sci. 14, 315-328 (2009).

5) Morita, T.; Fukuoka, T.; Imura, T.; Kitamoto, D. Production of glycolipid biosurfactants by basidiomycetous yeasts. Biotechnol. Appl. Biochem. 53, 39-49 (2009).

6) Imura, T.; Yanagishita, H.; Kitamoto, D. Coacervate formation from natural glycolipid: one acetyl group on the headgroup triggers coacervate-to-vesicle transition. J. Am. Chem. Soc. 126, 10804-10805 (2004).

7) Imura, T.; Ohta, N.; Inoue, K.; Yagi, H.; Negishi, H.; Yanagishita, H.; Kitamoto, D. Naturally engineered glycolipid biosurfactants leading to distinctive self-assembled structures. Chem. Eur. J. 12, 2434-2440 (2006).

8) Imura, T.; Hikosaka, Y.; Worakitkanchanakul, W.; Sakai, H.; Abe, M.; Konishi, M.; Minamikawa, H.; Kitamoto, D. Aqueous-phase behavior of natural glycolipid biosurfactants mannosylerythritol lipid A: Sponge, cubic, and lamella phases. Langmuir 23, 1659-1663 (2007).

9) Morita, T.; Kitagawa, M.; Suzuki, M.; Yamamoto, S.; Sogabe, A.; Yanagidani, S.; Imura, T.; Fukuoka, T.; Kita- 
moto, D. A yeast glycolipid biosurfactant, mannosylerythritol lipid, shows potential moisturizing activity toward cultured human skin cells: the recovery effect of MEL-A on the SDS-damaged human skin cells. $J$ Oleo Sci. 58, 639-642(2009).

10) Morita, T.; Konishi, M.; Fukuoka, T.; Imura, T.; Kitamoto, D. Discovery of Pseudozyma rugulosa NBRC 10877 as a novel producer of the glycolipid biosurfactants, mannosylerythritol lipids, based on rDNA sequence. Appl. Microbiol. Biotechnol. 73, 305-315 (2006).

11) Morita, T.; Konishi, M.; Fukuoka, T.; Imura, T.; Kitamoto, H.K.; Kitamoto, D. Characterization of the genus Pseudozyma by the formation of glycolipid biosurfactants, mannosylerythritol lipids. FEMS Yeast Res. 7, 286-292 (2007).

12) Heinrich, U.; Koop, U.; Leneveu-Duchemin, MC.; Osterrieder, K.; Bielfeldt, S.; Chkarnat, C.; Deqwert, J.; Hantschel, D.; Jaspers, S.; Nissen, HP.; Rohr, M.; Schneider, G.; Tronnier, H. Multicentre comparison of skin hydration in terms of physical-, physiological- and product-dependent parameters by the capacitive method (Corneometer CM 825). Int J Cosmet Sci. 25, 45-53 (2003).

13) Pinnagoda, J.; Tupker, R.; Agner, T.; Serup J.Guidelines for transepidermal water loss (TEWL) measurement. Contact Dermatitis. 22, 164-178(1990).

14) Wannasiri, W.; Imura, T.; Fukuoka, T.; Morita, T.; Sakai, H.; Abe, M.; Ratana, R.; Sumaeth, C.; Minamikawa, H.; Kitamoto, D. Phase behavior of ternary mannosyl- erythritol lipid/water/oil systems. Colloids and Surfaces B: Biointerfaces. 65, 106-112(2008).

15) Imokawa, G.; Hattori, M. A possible Function of Structural Lipids in the Water-Holding Properties of the Stratum Corneum. J Invest Dermatol. 84, 282-284 (1985).

16) Morita, T.; Takashima, M.; Fukuoka, T.; Konishi, M.; Imura, T.; Kitamoto, D. Isolation of basidiomycetous yeast Pseudozyma tsukubaensis and production of glycolipid biosurfactant, a diastereomer type of mannosylerythritol lipid-B. Appl. Microbiol. Biotechnol. 88, 679-688(2010).

17) Imokawa, G.; Abe, A.; Jin, K.; Higaki, Y.; Kawashima, M.; Hidano, A. Decreased Level of Ceramides in Stratum Corneum of Atopic Dermatitis: An Etiologic Factor in Atopic Dry Skin? J Invest Dermatol. 96, 523-526 (1991).

18) Jakob, J.; Lars, H.; Julie H.; Tue, D.; Gregor, J.; Tove A. Ceramides and Barrier Function in Healthy Skin. Acta Derm Venereol. 90, 350-353 (2010).

19) Imokawa, G.; Akasaki, S.; Hattori, M.; Yoshizuka, N. Selective Recovery of Deranged Water-Holding Properties by Stratum Corneum Lipids. J Invest Dermatol. 87, 758-761 (1986)

20) Fukuoka, T.; Morita, T.; Masaaki, K.: Imura, T.; Kitamoto, D. A basidiomycetous yeast, Pseudozyma tsukubaensis, efficiently produces a novel glycolipid biosurfactant. The identification of a new diastereomer of mannosylerythritol lipid-B. Carbohydr. Res. 25, 555560 (2008). 